\title{
Theoretical and numerical experiences on a test rig for active vibration control of mechanical systems with moving constraints
}

\author{
M. Rinchi* and E. Gambini \\ Dipartimento di Energetica 'S.Stecco' - Università di Firenze, Firenze, Italy
}

Questo Special Issue è per gli autori l'occasione per ricordare la gioia di vivere e la concretezza nel lavoro del Prof. Bruno Piombo. Il lavoro presentato in questo articolo si basa infatti su un'apparecchiatura sperimentale realizzata con il contributo del Ministero dell'Università e della Ricerca Scientifica MURST (ora MIUR) nell'ambito del programma COFIN 98 in un progetto di ricerca interuniversitario da Lui coordinato.

\begin{abstract}
Active control of vibrations in mechanical systems has recently benefited of the remarkable development of robust control techniques. These control techniques are able to guarantee performances in spite of unavoidable modeling errors. They have been successfully codified and implemented for vibrating structures whose uncertain parameters could be assumed to be time-invariant. Unfortunately a wide class of mechanical systems, such as machine tools with carriage motion realized by a ball-screw, are characterized by time varying modal parameters. The focus of this paper is on modeling and controlling the vibrations of such systems. A test rig for active vibration control is presented. An analytical model of the test rig is synthesized starting by design data. Through experimental modal analysis, parametric identification and updating procedures, the model has been refined and a control system has been synthesized.
\end{abstract}

\section{Introduction}

Active vibration control has gone through a notable acceleration in the last decades. Some of these methodologies are characterized by an intuitive form to whoever has a little competences in vibration mechanics (very useful in industrial practice) and on the other side they are well related to the developments of the modern robust control theories, such as LQG control (Linear Quadratic Gaussian) [5], $\mathrm{H}_{\infty}$ control [4], or VSC control (Variable Structure Control) [8]. For example IMSC technique (Independent Modal Space Control) [6,7] is widely used in industrial applications since it allows simple controller synthesis and implementation, and it assures at the same time high performances even with respect to quite large plant modelling uncertainties. These techniques mainly apply to mechanical systems whose dynamics is described by linear time-invariant differential equations (LTI systems).

However the case of machines whose dynamics is either non-linear and/or time varying is frequent. A wide class of dynamic systems is in fact characterized by differential linear equations whose coefficients vary with time.

For instance a NC machine tool moving the tool carriage through a ball-screw coupling is a typical example of LTV (Linear Time Varying) dynamic system. The unavoidable alignment error between screw and nut, also determine a characteristic system excitation: it has quite constant intensity and moves along the screw with the nut. This excitation determines generally little damped screw vibrations potentially dangerous for mechanical components

\footnotetext{
*Corresponding author: M. Rinchi, Dipartimento di Energetica 'S.Stecco', Via di S. Marta, 3 50139 Firenze (FI), Italy. Tel.: +39 55 4796 529; Fax: +39 554796 342; E-mail: rinchi@mapp1.de.unifi.it.
} 


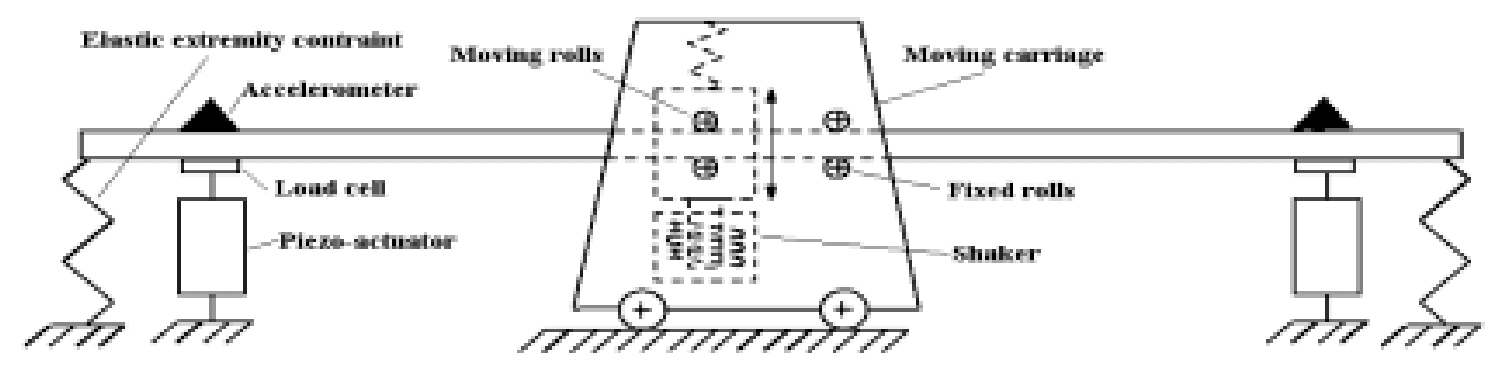

Fig. 1. Test rig sketch.

and detrimental for product quality (vibrations mean inaccuracy). The easier solution to limit screw vibrations is to reduce the speed of the machine tool, and the obvious negative consequence is the reduction of machine productivity.

In order to experimentally simulate the dynamics of these systems, a test rig has been realized in which a beam vibrates in the vertical plane, being characterized by a moving constraint and excitation. The main constitutive elements of the test rig are:

- a rectangular section beam elastically constrained at the ends vibrating only in the vertical plane;

- a carriage translating along the beam through a motor-reduction gear system. The mobile carriage realizes a moving constraint to the vertical translation of the screw. It is also equipped with an electromagnetic shaker that vertically excites the beam;

- two piezoelectric actuators, placed near the beam ends. They impose vibration control actions to the beam;

- two load cells placed between piezoelectric actuators and vibrating beam. They allow to realize local closed force loops;

- two piezoelectric accelerometers allowing real time vibration monitoring;

- a control system based on a DSP compatible with Matlab environment.

In Fig. 1 a sketch of the test rig is shown.

In Section 2 the rough model of the test rig, derived by design data, was refined by using constraints identification procedures. In Section 3 a more suitable model for control system design issues is presented, and in Section 4 its dynamic equations are derived. Starting by this model, in Sections 5 and 6 control system synthesis issues are discussed in order to control system damping. Particular attention is devoted in Section 7 to piezoactuators force control. Finally some simulation results are presented in Section 8.

\section{Constraints identification}

A rough mathematical model of the test rig was first derived. A FEM model of the beam was used, the 'first attempt' inertial properties of the excitation system and the stiffness of the fixed and mobile constraints were derived from design data. In order to refine this model 1, a number of experimental impact tests with an instrumented hammer have been carried out in order to find system Frequency Response Functions. Parametric identification techniques have been applied to these functions in order to refine the initial mathematical model [1]. Our attention has been mainly focused on the estimation of the elastic-inertial properties of both fixed and moving constraints, whose elements usually represent the main source of uncertainty affecting the nominal model the vibrating systems. Best-fit routines based on RFP (Rational Fraction Polynomial), IRFP (Iterative Rational Fraction Polynomial) and ILRFP (Iterative Logarithmic Rational Fraction Polynomial - a modification of IRFP method very useful near the antiresonances) are used to determine analytical expressions for system FRFs (in terms of fraction polynomials) at various positions assumed by the carriage. All these routines substantially consist on the least square minimization of a weighed error between experimental FRFs and analytical fixed-structure functions.

Starting from these analytical expressions for system FRFs, a further procedure (Inverse Sensitivity Method) was applied, in order to update elastic-inertial parameters of beam constraints. 
A FEM free-free model of the beam could be easily obtained from design data (we used the usual Timoshenko elements). If we denote mass and stiffness matrices of free-free system with $M_{\text {free-free }}$ and $\mathrm{K}_{\text {free-free }}$ respectively, we could express (total) constrained system matrices by assembling free-free matrices with those representing constraint contributions; system mass matrix may be then expressed by:

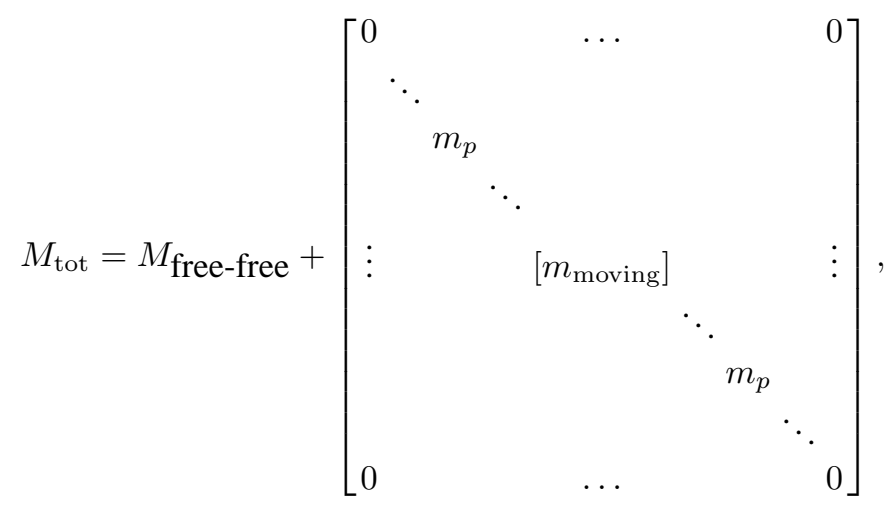

where:

$$
\left[M_{\text {moving }}\right]=\left[\begin{array}{cccc}
m_{s} & 0 & 0 & 0 \\
0 & 0 & 0 & 0 \\
0 & 0 & 0 & 0 \\
0 & 0 & 0 & 0
\end{array}\right]
$$

is the inertial carriage contribution, $m_{s}$ is the mass of moving excitation system and $m_{p}$ is the mass contribution due to each piezoelectric actuator (assumed to be equal).

Under the same assumptions stiffness matrix could be expressed as follows:

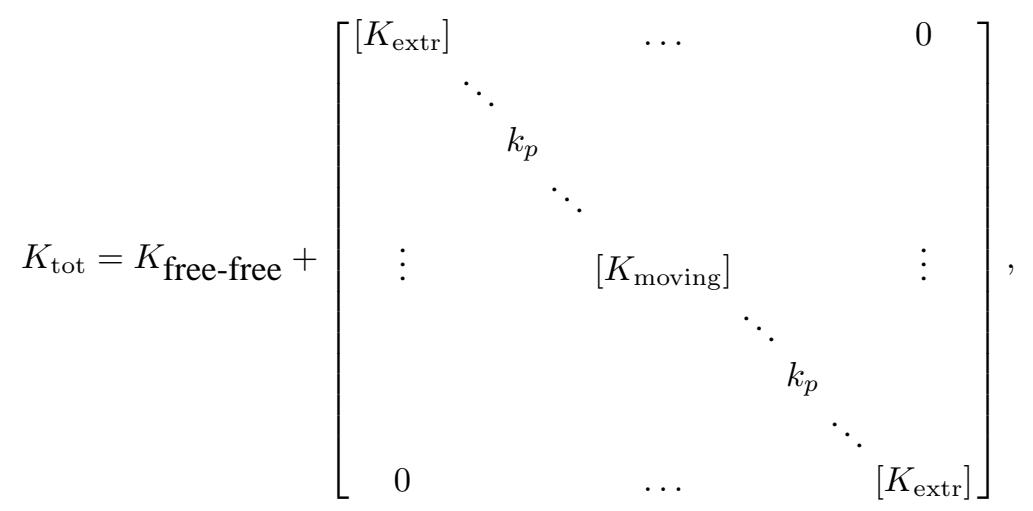

where:

$$
\left[K_{\text {extr }}\right]=\left[\begin{array}{ll}
k_{y y} & k_{y \varphi} \\
k_{\varphi y} & k_{\varphi \varphi},
\end{array}\right], \text { and }\left[K_{\text {moving }}\right]=\left[\begin{array}{cccc}
k_{s} & 0 & 0 & 0 \\
0 & 0 & 0 & 0 \\
0 & 0 & k_{r} & 0 \\
0 & 0 & 0 & 0
\end{array}\right]
$$

are the stiffness contributions due to the extremity constraints and the moving carriage respectively. $k_{y y}, k_{\varphi \varphi}, k_{y \varphi}$ are the translational, rotational and cross coupled stiffness of the extremity constraints (supposed to be equal at the two ends); $k_{p}$ the represents vertical translation stiffness due to piezoelectric actuators (assumed to be equal for both ones); $k_{r}$ is the vertical translation stiffness due to the couple of rolls realizing the mobile constraint, and $k_{s}$ is the vertical translation stiffness due to the elastic connection between the beam and the moving excitation system.

Denoting $\boldsymbol{v}$ the vector containing system resonances and antiresonances (in $0 \div 500 \mathrm{~Hz}$ frequency range) and $\boldsymbol{x}$ the vector containing the parameters to be identified, the relation mapping $\boldsymbol{x}$ to $\boldsymbol{v}(\mathrm{x})$ could be inverted by the knowledge 


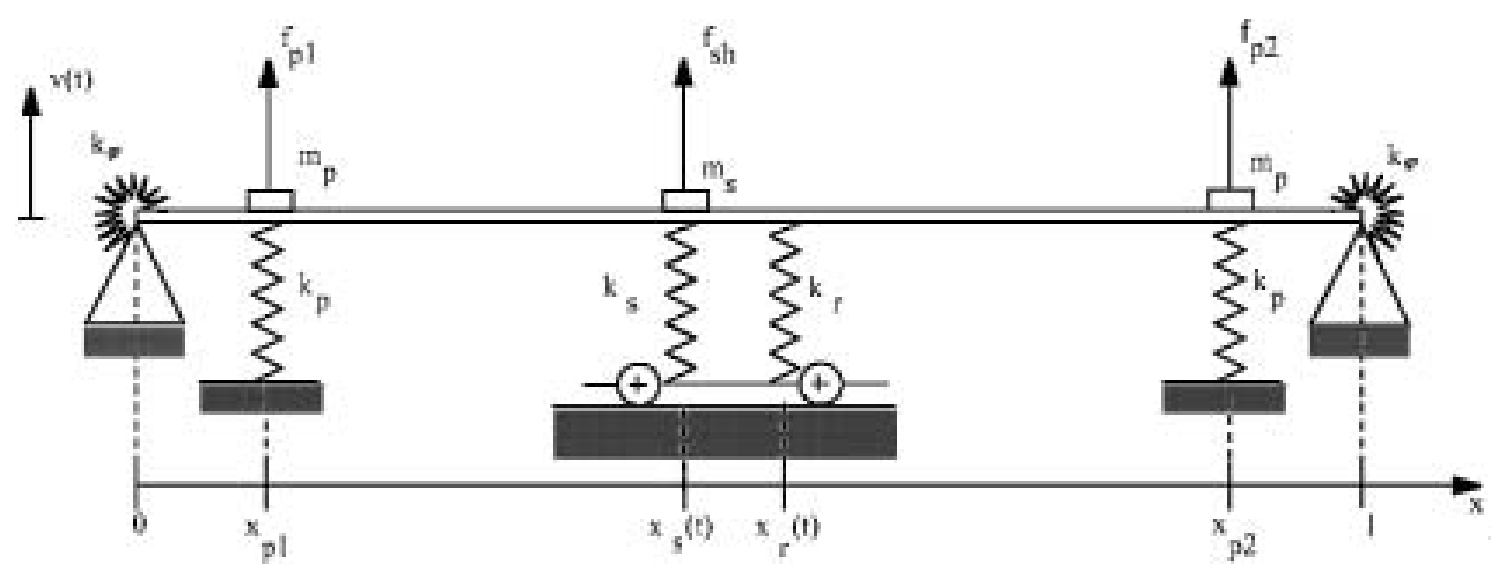

Fig. 2. Test rig mechanical model (vertical translations at ends are neglected).

of the experimental references for $\boldsymbol{v}$. Due to strong nonlinear relationship of $\boldsymbol{v}$ upon $\boldsymbol{x}$, constraint parameters identification is performed by the previously mentioned iterative approach, whose fundamental updating rule at step $r$ is the following:

$$
\boldsymbol{x}^{(r+1)}=\boldsymbol{x}^{(r)}+\left[\frac{\partial\left(\boldsymbol{W} \boldsymbol{v}_{a}\left(\boldsymbol{x}^{(r)}\right)\right)}{\partial \boldsymbol{x}}\right]\left(\left(\boldsymbol{W} v_{\exp }\right)-\left(\boldsymbol{W} \boldsymbol{v}_{a}\left(\boldsymbol{x}^{(r)}\right)\right)\right),
$$

where subscript $a$ stands for analytical, exp for experimental, and $\boldsymbol{W}$ is an opportune weighting matrix, chosen in such a way to find the solution minimizing the mean quadratic percentile error. The vector of the 'first attempt' values of unknown parameters $\boldsymbol{x}^{(0)}$ is derived from design data and iteration proceeded until satisfying convergence criteria involving both absolute and relative errors.

\section{Modeling for control system design}

Despite FEM model was successfully used for system identification, this has not been used for Control System Design. FEM model is in fact not much useful to describe test rig dynamics when the moving constraint effectively moves since the one to one correspondence between the position of the moving constraint and a node of the FEM model cannot be always assured. For this reason a new model was synthesised through the use of eigenfunctions technique. In order to easily model system dynamics, we assumed negligible the vertical displacements of the beam in correspondence of the extremity constraints (so $k_{y y}=k_{\varphi y}=k_{y \varphi}=\infty$ ). This simplifying assumption reveals very useful for model description in term of state-space equations and, on the other hand, it introduces negligible errors (this is also confirmed by the very high values of constraint stiffness resulting from model updating procedures). Furthermore we assumed the contributions of rotational inertia of beam sections to be negligible as well as the deformation effect of shearing stress.

The sketch of the resulting mechanical model of the test rig is Fig. 2.

The free vibrations of the beam - neglecting the presence of the moving carriage and of the force control system - may be analysed referring to the usual hinged-hinged Eulero-Bernoulli beam equation:

$$
\begin{aligned}
& \frac{\partial^{2} v}{\partial t^{2}}(x, t)+c^{2} \frac{\partial^{4} v}{\partial x^{4}}(x, t)=0 \\
& v(0, t) \equiv 0, v(l, t) \equiv 0, \quad \frac{\partial^{2} v}{\partial x^{2}}(0, t) \equiv 0, \quad \frac{\partial^{2} v}{\partial x^{2}}(l, t) \equiv 0,
\end{aligned}
$$

where we assumed:

$$
c=\sqrt{\frac{E J}{\rho S}}
$$


$E=$ Young modulus of the beam;

$J=$ beam cross section 2nd moment of area;

$S=$ beam cross section area;

$\rho=$ beam density.

It is well known that the solution of this equation leads to a Sturm-Liouville problem, and therefore to the generation of a set of orthogonal functions, commonly called eigenfunctions, each corresponding to a specific vibration frequency, called eigenvalue.

It is obvious that mode shapes and natural frequencies calculated in this way are different from those of system as illustrated in Fig. 2 (also considering the effects on system dynamics of carriage and piezoelectic actuators). Even though they are not the real system eigenfunctions, they have the very useful property to represent an orthogonal base of the space of regular functions defined in $x \in[0 ; l]$ respecting the essential (involving zero order derivative of displacement) boundary conditions. So we have that the vertical displacement $v$ of the elements of the beam, as a function of time $\mathrm{t}$ and the position $x$ along the beam, may be expressed as follows:

$$
v(x, t)=\sum_{k=1}^{\infty} q_{k}(t) \phi_{k}(x), \quad x \in[0, l], \quad t \geqslant 0,
$$

where:

$$
\phi_{k}(x)=\sin \left(k \pi \frac{x}{l}\right) .
$$

The previous expression may be approximated by limiting the summation to a finite number of addenda as follows:

$$
v(x, t)=\sum_{k=1}^{N} q_{k}(t) \phi_{k}(x)
$$

where the integer $N$ is sufficiently high. It is worth to note that, limiting the summation to a finite number $N$ of eigenfunctions, the natural boundary conditions $\left(v^{\prime \prime}(x, t)=-k_{\varphi \varphi}(E J)^{-1} v^{\prime}(x, t)\right.$ for $x=0$ and $\left.x=l\right)$ will be never exactly satisfied. This assumption obviously introduces an error in computation of potential elastic energy of the beam near its ends, nevertheless, since such error reduces as $N$ increases, we preferred to pay the price to involve a notable number of shape functions, but analytically very simple, rather than a limited number of very complicated shapes, as obtained from analysis of the beam provided with real boundary conditions. Evidently this approximation fits relatively small values of torsional stiffness $k_{\varphi \varphi}$. In the following sections, torsional stiffness will be simply referred as $k_{\varphi}$.

\section{System dynamics equations}

In order to get system dynamic equations, the Lagrange approach has been chosen. System kinetic energy $T$ has the following expression:

$$
T=\frac{1}{2}\left\{\int_{0}^{l} \rho A \dot{v}^{2}(x, t) d x+m_{p} \dot{v}^{2}\left(x_{p 1}, t\right)+m_{p} \dot{v}^{2}\left(x_{p 2}, t\right)+m_{s}\left[\dot{x}_{s}^{2}+\left(\dot{x}_{s} v^{\prime}\left(x_{s}(t), t\right)+\dot{v}\left(x_{s}(t), t\right)\right)^{2}\right]\right\},
$$

while the potential energy $V$ (neglecting the gravitational potential energy) may be expressed as follows:

$$
\begin{aligned}
V= & \frac{1}{2}\left\{\int_{0}^{l} E J v^{\prime \prime 2} d x+k_{p} v^{2}\left(x_{p 1}, t\right)+k_{p} v^{2}\left(x_{p 2}, t\right)+k_{s} v^{2}\left(x_{s}(t), t\right)+k_{r} v^{2}\left(x_{r}(t), t\right)+k_{\varphi} v^{\prime 2}(0, t)\right. \\
& \left.+k_{\varphi} v^{\prime 2}(l, t)\right\} .
\end{aligned}
$$

In the preceding expressions we put:

- $x_{p 1}$ and $x_{p 2}$ are the positions along the beam corresponding to the first (right) and the second (left) piezoelectric actuator respectively; 
- $x_{s}$ is the position along the beam corresponding to the moving excitation system;

- $x_{r}$ is the position along the beam corresponding to the moving constraint;

- $v^{\prime}(x, t)=\frac{\partial v(x, t)}{\partial x}$ is the rotation of the beam cross section in correspondence of the position $x$ along the beam.

If we put $L=T-V$, the differential equation governing the dynamics along the generic Lagrange coordinate may be calculated as follows:

$$
\frac{d}{d t} \frac{\partial L}{\partial \dot{q}_{k}}-\frac{\partial L}{\partial q_{k}}=Q_{k}, \quad k=1,2 \ldots, N,
$$

where:

$Q_{k}=Q_{k}(t)$ is the Lagrangian component of the non-conservative forces relative to the coordinate $q_{k}$.

In our case we have only concentrate non-conservative forces (not distributed), therefore we can express $Q_{k}$ as follows:

$$
Q_{k}=\sum_{i=1}^{n} f_{i}(t) \phi_{k}\left(x_{i}\right)
$$

where $f_{i}(t)$ is the force applied in correspondence of the point $x_{i}$, and $n$ is the total number of the forces acting on the system. Using modal coordinates, the boundary conditions of the beam may lead to the following expressions:

$$
\begin{aligned}
& \int_{0}^{l} \rho A \phi_{i}(x) \phi_{j}(x) d x=\hat{m}_{i} \delta_{i j} ; \\
& \int_{0}^{l} E J \phi_{j}^{\prime \prime}(x) \phi_{j}^{\prime \prime} d x=\hat{m}_{i} \hat{\omega}_{i}^{2} \delta_{i j} ;
\end{aligned}
$$

where $\hat{m}_{i}=\rho S \frac{l}{2}, \hat{\omega}_{i}=c\left(i \frac{\pi}{l}\right)^{2}$, and $\delta_{i j}=\left\{\begin{array}{l}0 \text { for } i \neq j \\ 1 \text { for } i=j\end{array}\right.$

Exploiting the previously mentioned properties of the eigensolutions, the system dynamic equations may be rewritten as follows:

$$
M(t) \ddot{\mathbf{q}}(t)+F(t) \dot{\mathbf{q}}(t)+K(t) \mathbf{q}(t)=B_{d} f_{s h}(t)+B_{u} \mathbf{u}(t)
$$

where $f_{s h}(t)$ represents the force applied to the beam by the electromagnetic exciter, and the vectors and matrices have the following expressions:

$$
\begin{aligned}
& \mathbf{q}(t)=\left[q_{1}(t), q_{2}(t), \ldots, q_{N}(t)\right]^{T} \\
& \mathbf{u}(t)=\left[\begin{array}{ll}
f_{p 1}(t) & f_{p 2}(t)
\end{array}\right]^{T}=\left[\begin{array}{ll}
-\theta V_{1} & -\theta V_{2}
\end{array}\right]^{T} ; \\
& {[M(t)]_{i j}=\hat{m}_{i} \delta_{i j}+m_{p} \phi_{i}\left(x_{p 1}\right) \phi_{j}\left(x_{p 1}\right)+m_{p} \phi_{i}\left(x_{p 2}\right) \phi_{j}\left(x_{p 2}\right)+m_{s} \phi_{i}^{\prime}\left(x_{s}(t)\right) \phi_{j}^{\prime}\left(x_{s}(t)\right) ;} \\
& {[F(t)]_{i j}=2 m_{s} \dot{x}_{s} \phi_{i}\left(x_{s}(t)\right) \phi_{j}^{\prime}\left(x_{s}(t)\right)} \\
& {[K(t)]_{i j}=m_{s} \ddot{x}_{s} \phi_{i}\left(x_{s}(t)\right) \phi_{j}^{\prime}\left(x_{s}(t)\right)+m_{s} \dot{x}_{s}^{2} \phi_{i}\left(x_{s}(t)\right) \cdot \phi_{j}^{\prime \prime}\left(x_{s}(t)\right)+\hat{\omega}_{i}^{2} \hat{m}_{i} \delta_{i j}+k_{p} \phi_{i}\left(x_{p_{1}}\right) \phi_{j}\left(x_{p 1}\right)} \\
& +k_{p} \phi_{i}\left(x_{p 2}\right) \phi_{j}\left(x_{p 2}\right)+k_{s} \phi_{i}\left(x_{s}(t)\right) \phi_{j}\left(x_{s}(t)\right)+k_{r} \phi_{i}\left(x_{r}(t)\right) \phi_{j}\left(x_{r}(t)\right)+k_{\varphi} \phi_{i}^{\prime}(0) \phi_{j}^{\prime}(0) \\
& +k_{\varphi} \phi_{i}^{\prime}(l) \phi_{j}^{\prime}(l) \\
& B_{d}(t)=\left[\phi_{1}\left(x_{s}(t)\right), \phi_{2}\left(x_{s}(t)\right), \ldots, \phi_{N}\left(x_{s}(t)\right)\right]^{T} ;
\end{aligned}
$$




$$
B_{u}=\left[\begin{array}{cc}
\phi_{1}\left(x_{p 1}\right) & \phi_{1}\left(x_{p 2}\right) \\
\vdots & \vdots \\
\phi_{N}\left(x_{p 1}\right) & \phi_{N}\left(x_{p 2}\right)
\end{array}\right] .
$$

In Eq. (10b) $V_{1}$ and $V_{2}$ are the input actuator voltages, while $\theta$ represents the force/voltage ratio with zero actuator deformation (see Eq. (13) below).

Once assigned the motion of the moving constraint (usually trapezoidal velocity profiles are used for the carriage), the terms $x_{s}, x_{r}, \dot{x}_{s}=\dot{x}_{r}, \ddot{x}_{s}=\ddot{x}_{r}$ become known functions, and the system acquires the typical characteristics of a FDLTV (Finite Dimensional Linear Varying with Time).

\section{Control system synthesis}

Numerical simulations have been performed imposing to the moving carriage a trapezoidal velocity profile, and to the shaker a force signal of the following type:

$$
f_{s h}=A_{s h} \sin \left(\frac{2 \pi}{P_{e q}}\left(x_{s}(t)-x_{s}(0)\right)\right) .
$$

This function represents the projection on the vertical plane of the interactions between screw and nut screw when the pitch is $p_{e q}$. Both simulation and experimental results show unacceptable beam vibrations for machining performances. Even these preliminary tests confirm that our system needs an effective active vibration control system to efficiently work.

\section{Active vibrations damping}

In performing system identification (when the carriage was still) practical remarks have pointed out in order to guarantee system stability. In order to oppose the so-called spillover phenomenon, that is the destabilizing effect of the high frequencies dynamics (neglected in the nominal model) on the real system control loop [2], the use of co-located actuators and sensors (placed in the same position) was suggested.

Nevertheless the high system stiffness near piezoelectric actuators (piezoactuator-load cell series are very stiff, and furthermore they must be placed near extremity constraint to allow carriage maximum mobility) involves problems of system observability and controllability. For this reason it is difficult to obtain an efficient control action by elaborating these system outputs. As a preliminary approach, we suppose to neglect actuators dynamics (analytically imposing $m_{p}=k_{p}=0$ ) obtaining great benefits for that concerning system observability. In such hypothesis we investigated the performances of the following simple control law:

$$
\left[\begin{array}{l}
f_{p 1} \\
f_{p 2}
\end{array}\right]=-C_{v}(t)\left[\begin{array}{l}
\dot{v}\left(x_{p 1}\right) \\
\dot{v}\left(x_{p 2}\right)
\end{array}\right] ;
$$

where $C_{v}(t)$ is the only design parameter. The use of the control law expressed by Eq. (12), that is actually a simple velocity feedback, allows practically to model piezoactuators as viscous dampers in which damping coefficient varies with time in order to get the best vibration suppression performance. The control action is power-consuming, and therefore surely stabilizing. The velocities $\dot{v}\left(x_{p 1}\right)$ and $\dot{v}\left(x_{p 2}\right)$ of the beam at the actuators' positions may be obtained by discrete-time integration of the signals coming by the accelerometers.

Various criteria could be used to determine suitable $C_{v}(t)$ law; in this paper we choose to make $C_{v}$ depending on the position of the moving carriage along the beam (gain scheduling). For each position of the carriage, we consider our system to be LTI (Linear Time Invariant) so that its dynamics, for a given $x_{r}$, may be determined by introducing the (constant) values of $x_{s}$ and $x_{r}$ in system dynamic equations. The feedback gain value is chosen so as to maximize beam first mode damping factor. Feedback gains have been calculated for a discrete number of carriage positions. The values to be applied when the carriage is in intermediate positions are estimated through linear interpolation.

It is important to point out that this approach may be exploited also in the case in which the carriage velocity profile is not known a priori: the position of the carriage (the scheduling parameter) may be in fact instantaneously measured by the resolver of the servoactuator moving the carriage. 


\section{Actuators force control}

In the preceding paragraph we assumed to be able to exactly regulate the interaction force between the beam and the piezoactuators: even though the control law is very simple, the reduction of the flexural oscillations of the beam is significant. Unfortunately the above mentioned assumption is not always acceptable when control actuators are of the piezoelectric type. These actuators in fact allow an easy system regulation in position, but they are not useful for force control.

The (electromechanical) dynamic equation of each piezoactuator may be written as follows:

$$
-m_{p} \ddot{v}-k_{p} v-\theta V=\hat{f}_{p}
$$

where $\theta$ is the actuator expansion coefficient, $V$ is the input actuator tension, and $\hat{f}_{p}$ is the force effectively applied to the beam. Upstream of each actuator we should also consider the presence of the voltage amplifier: as well known, also the amplifier has its dynamics, and it may be approximated by a first order low-pass filter:

$$
G(s)=\frac{L[V(t)]}{L\left[V_{c}(t)\right]}=\frac{A_{\mathrm{amp}}}{\tau s+1}
$$

where $L$ stands for Laplace operator, $V(t)$ is the input actuator tension (that is output amplifier tension), $V_{c}$ is the control voltage (that is the input signal for each amplifier), $A_{\mathrm{amp}}$ is the static gain, $\tau=\frac{1}{2 \pi f_{\mathrm{cut}}}$ is the time constant, and $f_{\text {cut }}$ is the cut-off frequency corresponding to $-3 \mathrm{~dB}$ response.

The amplifier-actuator series represent a new system to be controlled, whose dynamics may be described in terms of state-space equations as follows:

$$
\left\{\begin{array}{l}
\dot{x}=-\frac{1}{\tau} x-\frac{\theta A_{\mathrm{amp}}}{\tau} V_{c} \\
\hat{f}_{p}=x+d
\end{array}\right.
$$

where $d=-m_{p} \ddot{v}-k_{p} v$ represents the disturbances to the force output signal, depending on the elastic-inertial characteristics of the actuators.

The load cells placed between each actuator and the beam measure the force applied to the beam; these signals are the inputs for the force control system. They are compared with the reference signals - called force setpoints and consequently the controller generates voltage signals to be fed to the actuators in order to minimize the error. It is obvious that we need an effective disturbance rejection to make the real force to follow the setpoint. A class of control systems that well satisfies our demands is that of the $S M C$ controller (Sliding Mode Control). Using the procedure described in [3] the following control law has been identified:

$$
V_{c}=-\frac{\tau}{\theta A_{\mathrm{amp}}}\left(\dot{r}+\frac{1}{\tau} \hat{f}_{p}+\psi \operatorname{sign}(e)\right) ;
$$

where:

$r$ is the force setpoint;

$e=r-\hat{f}_{p}$ is the error signal;

$\psi>\left(1+\max \left(\left|\dot{d}+\frac{d}{\tau}\right|\right)\right)$ is a design constant.

By analysing the previous equations it is immediate to verify that the error dynamics in each force closed loop satisfies the following condition:

$$
\frac{1}{2} \frac{d}{d t}\left(e^{2}\right)<-|e|
$$

usually known as sliding condition.

In the analytical expression of the control law it is important to notice the presence of the feedforward term $\dot{r}$ : it is well known that real-time derivation of a signal often involves very large errors. Despite this may seem a weak point of this control strategy, in our system it doesn't represent a real problem. By calculating the analytical expression of the feedforward term we obtain:

$$
\dot{r}=\frac{d}{d t}\left(-C_{v}\left(x_{r}(t)\right) \dot{v}\right)=-\left.\frac{d C_{v}}{d x}\right|_{x_{r}} \dot{x}_{r} \dot{v}-C_{v}\left(x_{r}(t)\right) \ddot{v} ;
$$


being $C_{v}\left(x_{r}\right)$ a known function and the acceleration signal directly measured by the accelerometers co-located with the actuator, it is in fact unnecessary to implement any real-time differentiation. The integration of the control strategies Eqs (16) and (18) (velocity feedback and SMC) leads to a decentralized control scheme consisting, for each actuator, in the following feedback law:

$$
V_{c}=-\frac{\tau}{\vartheta A_{\mathrm{amp}}}\left(-\left.\frac{d C_{v}}{d x}\right|_{x_{r}} \dot{x}_{r} \dot{v}-C_{v}\left(x_{r}(t)\right) \ddot{v}+\frac{1}{\tau} \hat{f}_{p}+\psi \cdot \operatorname{sign}(e)\right) .
$$
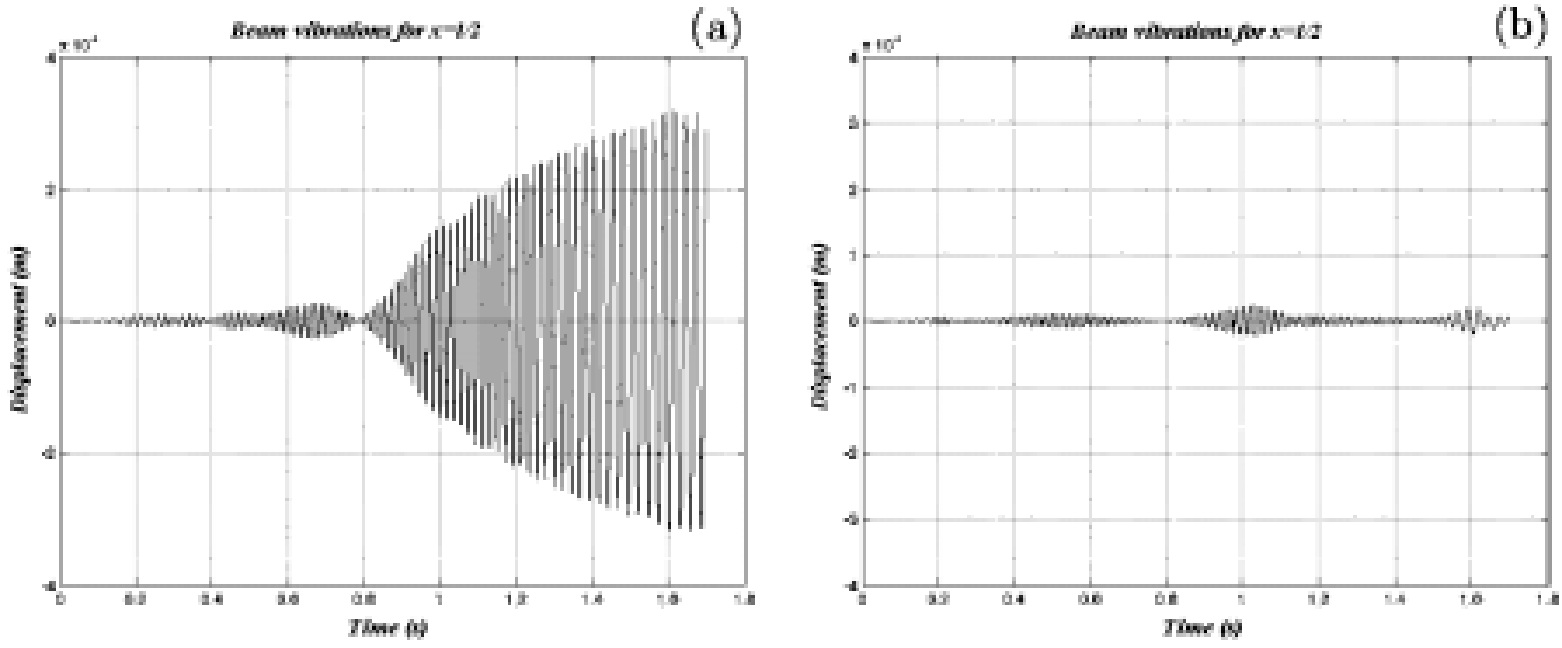

Fig. 3. Beam half-point vibrations: a) open loop, b) closed loop.

\section{Simulation results}

In order to test control strategy effectiveness, various simulations have been carried out. All system parameters used for simulation are reported in Table1, in which we evidenced the design data and those resulting by the use of
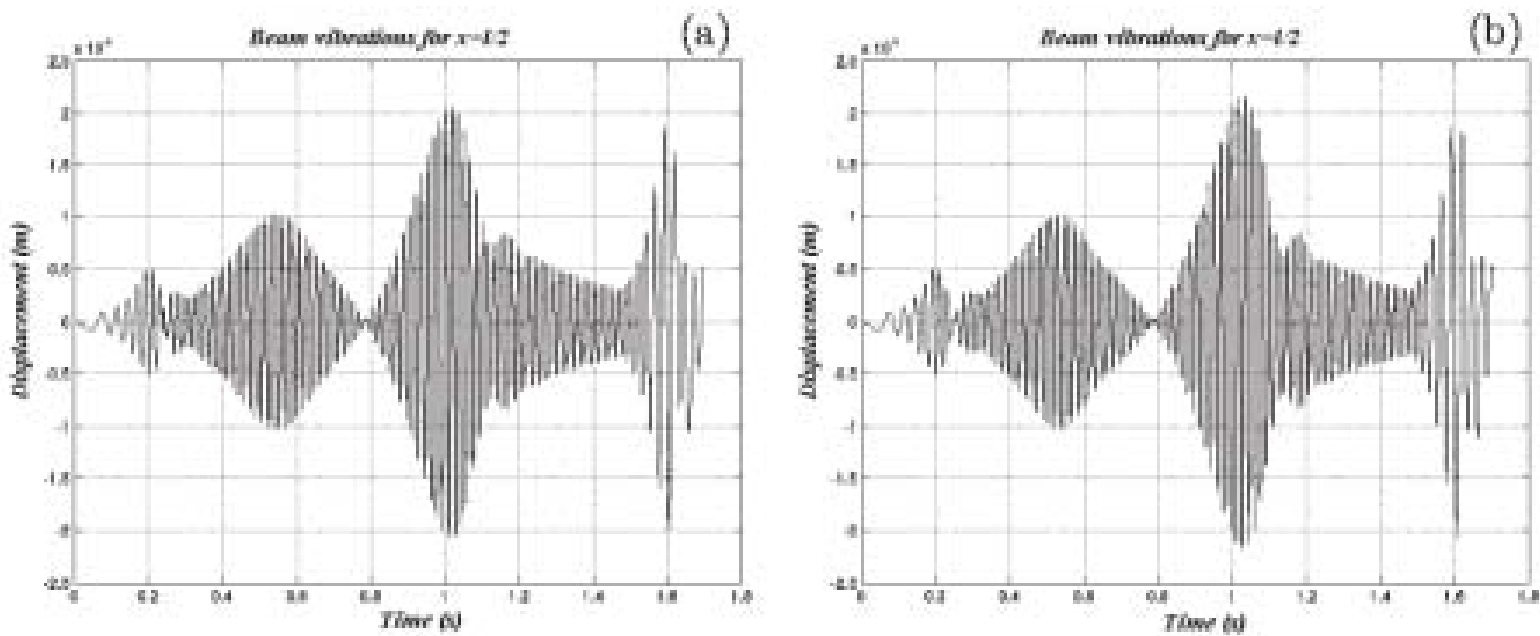

Fig. 4. Beam half-point vibrations: a) 'ideal' force control, b) SMC force controller. 
the previously mentioned system identification procedures (starting from experimental data). All simulations were performed assuming ten modal shapes $(N=10)$. The first step was the simulation of the closed loop dynamics assuming the force control to be ideal and implementing the gain scheduling feedback law Eq. (12). In particular the velocity feedback gains maximizing the first mode damping have been calculated for eight configurations, i.e. for eight positions of the carriage equally spaced along the beam (their values are reported in Table 1). When the carriage is at an intermediate position between two of these configurations, the velocity feedback gain is calculated through linear interpolation. Figure 3 describes beam half-point oscillations in open loop (a) and closed loop (b), demonstrating an effective reduction of vibrations. The second step was the simulation of the 'real' control system relying on inner sliding mode control loops on actuators - in order to test its performance and to compare them with those of the 'ideal' control system. In Fig. 4(a) the vibrations of the beam half point were plotted assuming the force control system to be ideal (the same of Fig. 3). In Fig. 4(b) the vibrations of the same point are plotted when using the control law Eq. (19). In Fig. 5 the above mentioned vibrations of beam half-point are translated in frequency domain. Simulation results show that beam vibrations are substantially identical.

Table 1.

System parameters used for numeric simulations

\begin{tabular}{|c|c|c|c|c|c|c|c|c|c|c|c|c|c|c|}
\hline \multirow{2}{*}{$\begin{array}{c}\text { design } \\
\text { data }\end{array}$} & $\begin{array}{c}l \\
(\mathbf{m})\end{array}$ & \multicolumn{2}{|c|}{$\begin{array}{c}S \\
\left(m^{2}\right)\end{array}$} & \multicolumn{2}{|c|}{$\begin{array}{c}E \\
\left(\mathrm{~N} / \mathbf{m}^{2}\right)\end{array}$} & \multicolumn{2}{|c|}{$\begin{array}{c}\rho \\
\left(\mathrm{kg} / \mathrm{m}^{3}\right)\end{array}$} & \multicolumn{2}{|c|}{$\begin{array}{c}J \\
\left(\mathbf{m}^{4}\right) \\
\end{array}$} & \multicolumn{2}{|c|}{$\begin{array}{c}\theta \\
\text { (N/Volt) }\end{array}$} & $\begin{array}{l}x_{p 1} \\
(\mathbf{m})\end{array}$ & \begin{tabular}{|l|}
$x_{p 2}$ \\
$(\mathrm{~m})$ \\
\end{tabular} & $\begin{array}{c}x_{r-} x_{s} \\
(\mathbf{m})\end{array}$ \\
\hline & 1 & \multicolumn{2}{|c|}{$2.4 \cdot 10^{-4}$} & \multicolumn{2}{|c|}{$2.06 \cdot 10^{11}$} & \multicolumn{2}{|c|}{$7.8 \cdot 10^{3}$} & \multicolumn{2}{|c|}{$7.2 \cdot 10^{-10}$} & \multicolumn{2}{|c|}{-14.4} & .03 & .97 & \multirow{2}{*}{$\begin{array}{c}0.075 \\
\mathbf{k}_{\mathbf{r}} \\
(\mathbf{N} / \mathbf{m})\end{array}$} \\
\hline \multirow{2}{*}{$\begin{array}{c}\text { identified } \\
\text { data }\end{array}$} & \multicolumn{2}{|c|}{$\begin{array}{c}\mathbf{m}_{\mathbf{s}} \\
(\mathrm{kg})\end{array}$} & \multicolumn{2}{|c|}{$\begin{array}{c}m_{p} \\
(\mathrm{~kg})\end{array}$} & \multicolumn{2}{|c|}{$\begin{array}{c}\mathbf{k}_{\mathrm{yy}} \\
(\mathrm{N} / \mathbf{m})\end{array}$} & \multicolumn{2}{|c|}{$\begin{array}{c}\mathbf{k}_{\mathrm{y} \varphi} \\
(\mathrm{N} / \mathbf{r a d})\end{array}$} & \multicolumn{2}{|c|}{$\begin{array}{c}\mathbf{k}_{\varphi \varphi} \\
(\mathrm{N} \cdot \mathbf{m} / \mathbf{r a d})\end{array}$} & \multicolumn{2}{|c|}{$\begin{array}{c}k_{\mathrm{p}} \\
(\mathrm{N} / \mathbf{m})\end{array}$} & $\begin{array}{c}\mathbf{k}_{\mathrm{s}} \\
(\mathrm{N} / \mathbf{m})\end{array}$ & \\
\hline & \multicolumn{2}{|c|}{1.751} & \multicolumn{2}{|c|}{$1.13 \cdot 10^{-2}$} & \multicolumn{2}{|c|}{$1.06 \cdot 10^{8}$} & \multicolumn{2}{|c|}{$5.589 \cdot 10^{5}$} & \multicolumn{2}{|c|}{$4.48 \cdot 10^{3}$} & \multicolumn{2}{|c|}{$16 \cdot 10^{6}$} & 248 & $2.002 \cdot 10^{8}$ \\
\hline carriage & \multicolumn{5}{|c|}{$0<t<0.2273$} & \multicolumn{5}{|c|}{$0.2273<\mathrm{t}<1.4727$} & & \multicolumn{3}{|c|}{$1.4727<\mathrm{t}<1.7$} \\
\hline $\begin{array}{c}\text { law } x_{r}(t) \\
(\mathrm{m})\end{array}$ & \multicolumn{5}{|c|}{$1.1 \cdot \mathrm{t}^{2}+0.1636$} & \multicolumn{5}{|c|}{$0.5 \cdot t+0.1068$} & & \multicolumn{3}{|c|}{$-1.1 \cdot \mathrm{t}^{2}+3.74 \cdot \mathrm{t}-2.279$} \\
\hline amplifier & & amp & & $\begin{array}{c}\tau \\
\text { (s) }\end{array}$ & & & sturba & ince & & $\begin{array}{l}A_{s h} \\
(\mathrm{~N})\end{array}$ & & & $\begin{array}{l}p_{e \theta} \\
(\mathbf{m}\end{array}$ & \\
\hline & & 10 & & .003 & & & & & & 1 & & & .00 & \\
\hline velocity & $\overline{\underline{x_{r}}=}$ & 0.1 & & 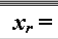 & 0.269 & & $\underline{\underline{c_{r}=0 .} .}$ & 374 & & $=0.479$ & & & & \\
\hline feedback & & 5950 & & & 565 & & $170^{\prime}$ & & & 5557 & & gain & & \\
\hline$C_{v}\left(x_{\nu}\right)$ & $x_{r}=$ & 0.5 & & $x_{r}=$ & 0.690 & & $c_{r}=0$. & 795 & & $=0.9$ & & $\psi$ & & 10000 \\
\hline$(\mathrm{N} \cdot \mathrm{s} / \mathrm{m})$ & & 7074 & & & 565 & & $247^{\prime}$ & & & 7649 & & & & \\
\hline
\end{tabular}
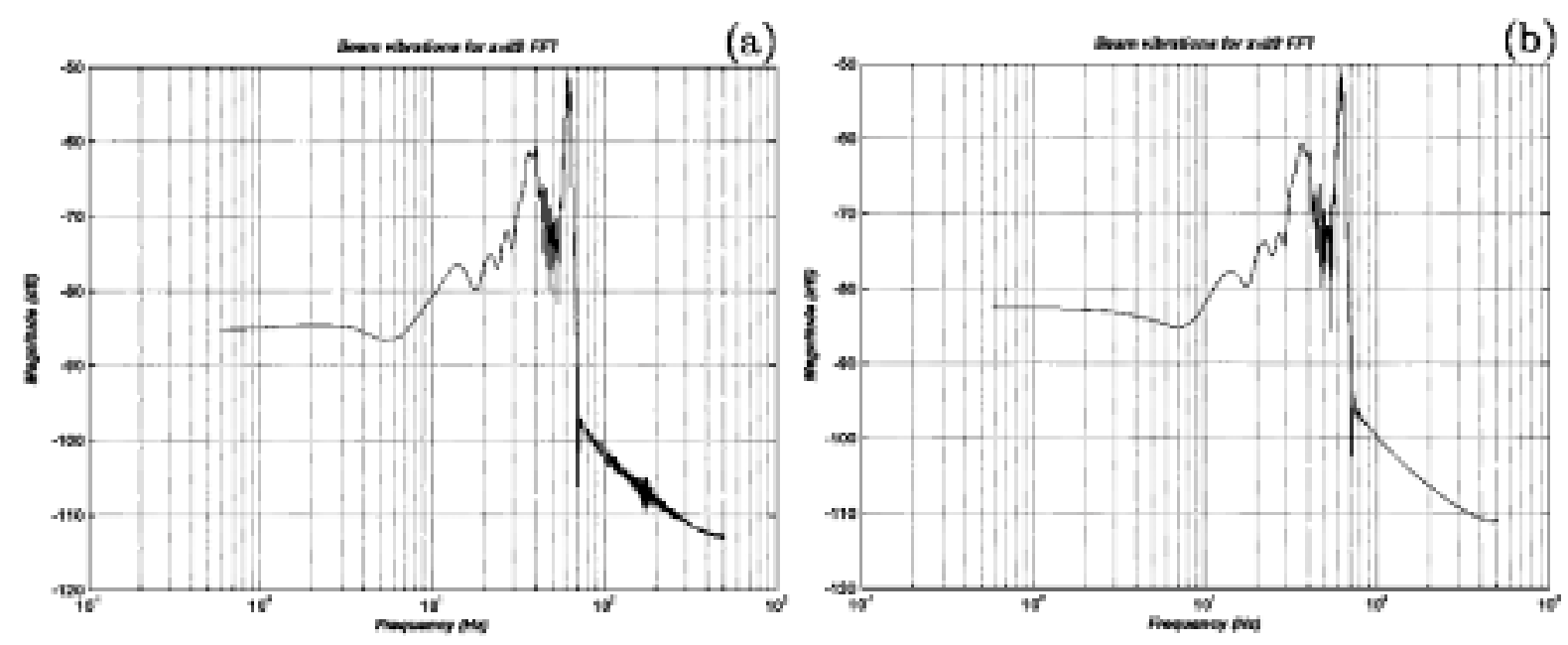

Fig. 5. Frequency spectrum of beam half-point vibrations: a) 'ideal' force control, b) SMC force controller. 


\section{Conclusions}

Active control of vibrations in flexible systems with time-varying dynamics still represent a research field not fully explored. The test rig presented in this paper allows the experimentation of active control techniques, and the one we presented has the advantage to be easily implementable and well fitting accelerometric sensors and piezoelectric actuators. In this paper we have also mentioned modeling and system parametrical identification procedures we believe very useful for control system synthesis. Even if simulation results are quite encouraging, some preliminary experimental tests have been carried out to verify the control system performance. Some problems have been found in the implementation of the damping control due to the difficult extraction of appreciable signals from the accelerometers co-located with the piezoactuators. In this configuration both actuators and sensors must be in fact placed very close to the extremity constraints (and so beam nodes), and that implies relatively small accelerations and an high value of noise-to-signal ratio. Currently some modifications to test rig hardware and measurement chains are scheduled in order to solve these problems.

\section{References}

[1] B. Allotta, F. Angioli and M. Rinchi, Constraints Identification for Vibration Control of Time-Varying Boundary Conditions Systems, Proc. of IEEE/ASME International Conference on Advanced Intelligent Mechatronics, Como, 2001, Vol. 1, 606-611.

[2] A. Cavallo, G. De Maria, E. Leccia and R. Setola, A Robust Controller for active vibration control of flexible systems, In Proc. of 36th IEEE Conference on Decision \& Control, San Diego, 1997, Vol. 2, 1355-1360.

[3] R.A. De Carlo, S.H. Zak and G.P. Matthews, Variable Structure Control of Nonlinear Multivariable Systems: a Tutorial, Proc. of the IEEE 76(3) (1988), 212-232.

[4] B.A. Francis, A Course in $H_{\infty}$ Control Theory, Springer-Verlag, Berlin, 1987.

[5] H. Kwakernaak and R. Sivan, Linear Optimal Control Systems, Wiley-Interscience, New York, 1972.

[6] L. Meirovitch and L. Silverberg, Control of Structures Subjected to Seismic Excitation, J. of Civil Engineers 109(2) (1983), 604-616.

[7] H. Öz and L. Meirovitch, Optimal Control of Flexible Gyroscopic Systems, J. of Guidance 3 (1980), 218-226.

[8] V.I. Utkin, Sliding Modes and their Application in Variable Structure Control, MIR, Moscow, 1978. 

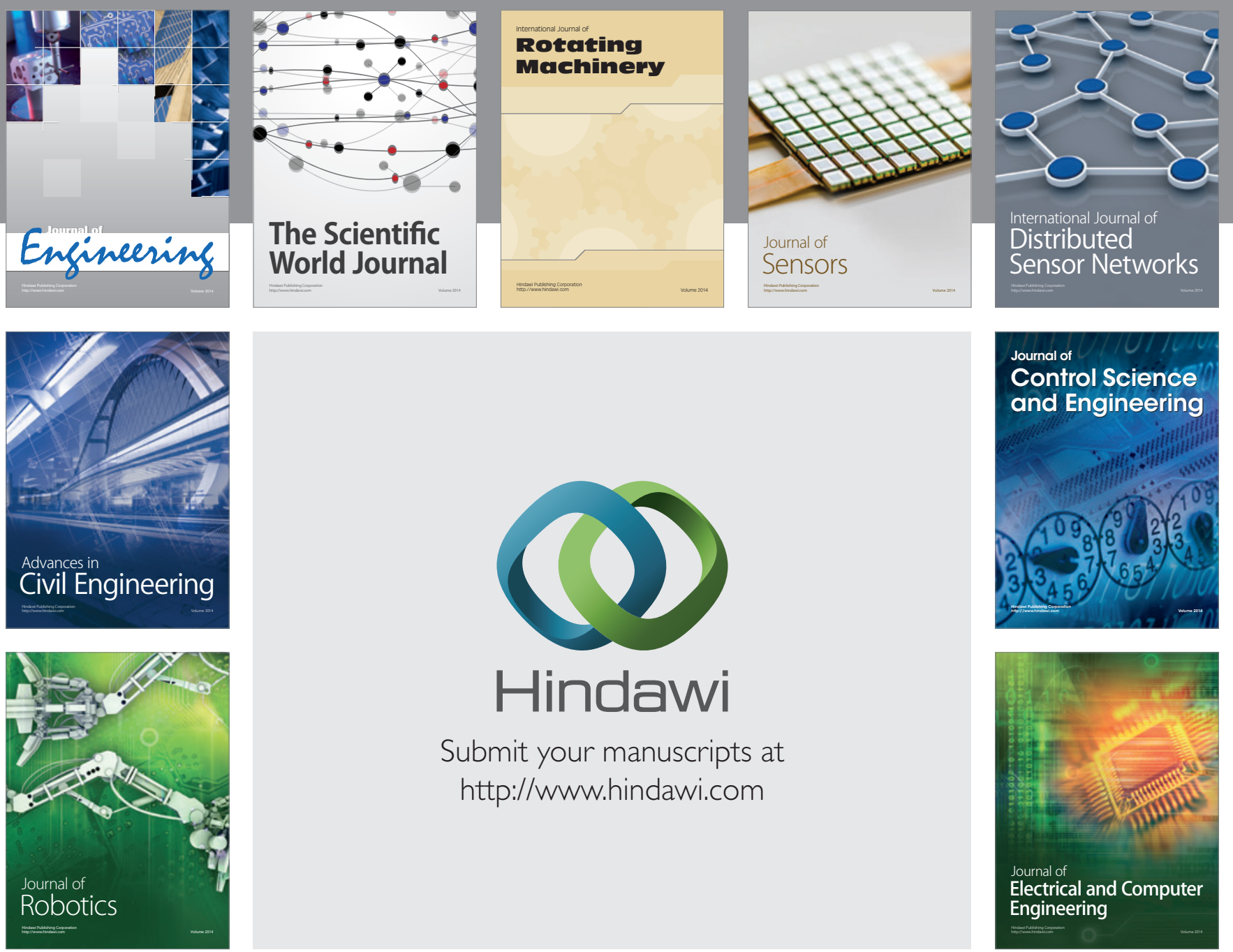

Submit your manuscripts at

http://www.hindawi.com
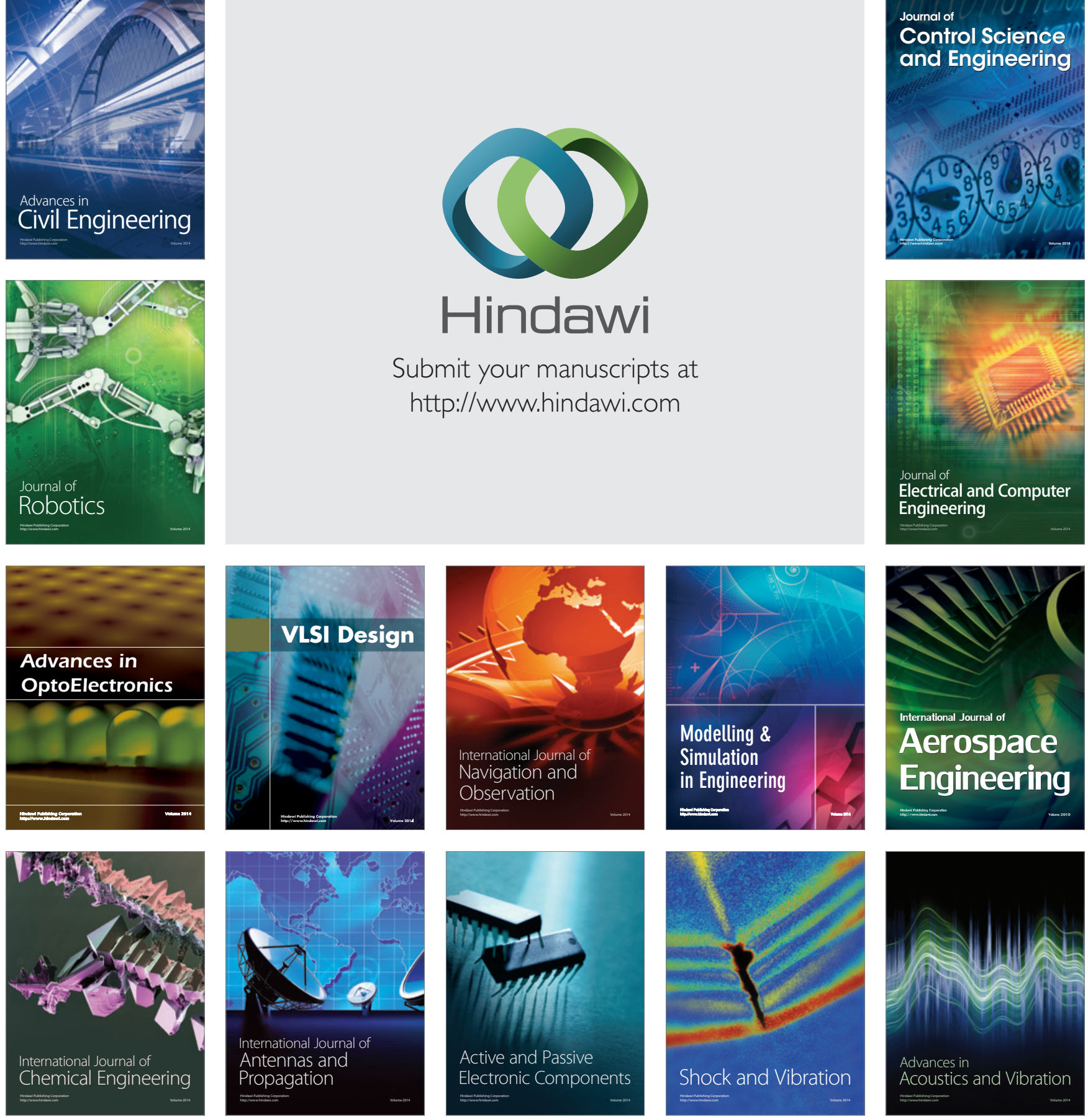